\title{
Safety and Efficacy of Pirfenidone in Advanced Idiopathic Pulmonary Fibrosis: A Nationwide Post- Marketing Surveillance Study in Korean Patients
}

\author{
Man Pyo Chung - Moo Suk Park - In Jae Oh · Heung Bum Lee • \\ Young Whan Kim · Jong Sun Park · Soo Taek Uh • Yun Seong Kim • \\ Yangjin Jegal · Jin Woo Song
}

Received: February 29, 2020 / Published online: April 15, 2020

(C) The Author(s) 2020

\section{ABSTRACT}

Aim: The efficacy and safety of pirfenidone have been previously demonstrated in patients with mild-to-moderate idiopathic pulmonary fibrosis (IPF). However, the effect of pirfenidone in patients with advanced IPF remains unclear.

Digital Features To view digital features for this article go to https://doi.org/10.6084/m9.figshare.12039624.

Electronic supplementary material The online version of this article (https://doi.org/10.1007/s12325020-01328-8) contains supplementary material, which is available to authorized users.

\section{P. Chung}

Division of Pulmonary and Critical Care Medicine, Department of Medicine, Samsung Medical Center, Sungkyunkwan University School of Medicine, Seoul, South Korea

\section{S. Park}

Division of Pulmonary and Critical Care Medicine, Department of Internal Medicine, Yonsei University College of Medicine, Yonsei University Health System, Seoul, South Korea

I. J. Oh

Department of Internal Medicine, Lung and Esophageal Cancer Clinic, Chonnam National University Medical School, Hwasun, South Korea

H. B. Lee

Department of Internal Medicine, Research Institute of Clinical Medicine, Medical School of Jeonbuk National University, Jeonju, South Korea
Here, we investigated the effects of pirfenidone against advanced IPF in a real-world setting. Methods: A prospective nationwide post-marketing study was conducted on 258 patients from 10 Korean institutions. Patients with a predicted forced vital capacity (FVC) less than $50 \%$ or a diffusing capacity of the lung for carbon monoxide (DLco) less than 35\% at baseline were classified as the advanced IPF group.

Results: Of 219 patients included in the analysis, the majority were male $(76.3 \%)$; the mean age was 67.3 years, and the advanced group

\section{Y. W. Kim}

Division of Pulmonary and Critical Care Medicine, Department of Internal Medicine, Seoul National University Hospital, Seoul, South Korea

\section{J. S. Park}

Division of Pulmonary and Critical Care Medicine, Department of Internal Medicine, Seoul National University Bundang Hospital, Seongnam, South Korea

\section{S. T. Uh}

Division of Allergy and Respiratory Medicine, Department of Internal Medicine, Soonchunhyang University Seoul Hospital, Seoul, South Korea

\section{Y. S. Kim}

Department of Internal Medicine, Pusan National University Yangsan Hospital, Yangsan, South Korea 
accounted for $17.8 \%$ of the patients. The median treatment duration was 298 days. Among the subjects, $86.3 \%$ experienced adverse events (AEs), of which a decreased appetite (32.4\%) and a photosensitivity reaction $(13.7 \%)$ were the most frequent. The incidence of AEs was similar between the advanced and non-advanced groups $(92.3 \%$ vs. $85.0 \%$, respectively; $p=0.229$ ). Although the overall discontinuation rate was higher in the advanced group than in the non-advanced group $(74.4 \%$ vs. $50.0 \%$, respectively; $p=0.006$ ), the percentages of the patients who discontinued treatment as a result of AEs were similar in both groups $(20.5 \%$ vs. $23.3 \%$, respectively; $p=0.704$ ). In all patients, the rates of decline in the predicted FVC and DLco over 48 weeks were $-4.3 \pm 1.3 \%$ and $-4.4 \pm 1.7 \%$, respectively. There was no between-group difference in the rate of lung function decline.

Conclusions: Pirfenidone used for the treatment of patients with IPF in a real-world setting was well tolerated, with an acceptable safety profile and a consistent therapeutic effect, regardless of the disease severity.

Trial Registration: ClinicalTrials.gov NCT03761082; the trial was retrospectively registered on December 3, 2018.

Keywords: Advanced disease; Disease progression; Idiopathic pulmonary fibrosis; Pirfenidone; Safety; Treatment outcome

Y. Jegal

Division of Pulmonary Medicine, Department of Internal Medicine, Ulsan University Hospital, University of Ulsan College of Medicine, Ulsan, South Korea

J. W. Song $(\varangle)$

Department of Pulmonary and Critical Care Medicine, Asan Medical Centre, University of Ulsan College of Medicine, Seoul, South Korea

e-mail: jwsongasan@gmail.com

\section{Key Summary Points}

Why carry out this study?

The efficacy and safety of pirfenidone have been previously demonstrated in patients with mild-to-moderate idiopathic pulmonary fibrosis (IPF), but the effect of pirfenidone in patients with advanced IPF remains unclear.

Several real-world studies have suggested that pirfenidone may attenuate lung function decline in patients with advanced IPF, but these studies were mostly retrospective observational, singlecenter, or descriptive studies.

We investigated the effects of pirfenidone against advanced IPF in a real-world setting.

\section{What was learned from the study?}

Treatment with pirfenidone in a realworld setting produced an acceptable safety profile and a consistent therapeutic effect in patients with IPF in terms of stabilizing lung function and subjective symptoms, regardless of the disease severity.

Although the demographic and clinical features of the present study were comparable to those of other studies, additional studies may be needed to further confirm these findings.

\section{INTRODUCTION}

Idiopathic pulmonary fibrosis (IPF) is a fatal fibrotic lung disease, associated with the worsening of dyspnea and a progressive decline in lung function, with a median patient survival of $2-5$ years from diagnosis $[1,2]$. Pirfenidone, an oral antifibrotic agent [3], was approved for IPF treatment in several countries, including Japan, European countries, and the USA. The drug has 
been commercially available in Korea since October 2012.

Previous clinical trials have shown that treatment of patients with IPF with pirfenidone attenuates lung function decline and reduces the risk of unexpected hospitalizations and mortality, with the drug showing an acceptable safety profile [4-9]. A post hoc analysis of pooled data from three phase 3 trials [10] also showed consistent benefits of pirfenidone for lung function in patients with IPF, regardless of the extent of lung function impairment [forced vital capacity (FVC) at least $80 \%$ vs. less than $80 \%$ ] or the Gender-Age-Physiology stage (I vs. II-III). However, previous trials only included patients with mild-to-moderate IPF [predicted FVC at least $50 \%$ and predicted diffusing capacity of the lung for carbon monoxide (DLco) at least 35\%], and thus, the effect of pirfenidone in patients with advanced IPF remains unclear.

Several real-world studies and post hoc analyses of the RECAP (NCT00662038) trial have suggested that pirfenidone may attenuate lung function decline in patients with advanced IPF [11-15]. However, these studies were mostly retrospective observational [11-15], single-center $[11,12]$, or descriptive studies [15]. Therefore, the aim of this study was to investigate the effect of pirfenidone treatment in patients with advanced IPF compared with that in patients with non-advanced IPF in a real-world setting.

\section{METHODS}

\section{Patients}

Between 2014 and 2017, 258 patients with IPF were enrolled from 10 institutions in Korea and followed up until treatment discontinuation or the end of the study period. The diagnosis of IPF was confirmed through multidisciplinary discussion at each site, based on the American Thoracic Society (ATS)/European Respiratory Society (ERS)/Japanese Respiratory Society/Latin American Thoracic Association guidelines [1]. According to the package insert statement, an initial dose of $200 \mathrm{mg}$ of pirfenidone was administered three times daily with meals
(600 $\mathrm{mg} /$ day). Thereafter, the dose was gradually increased by $200 \mathrm{mg}$ every 2 weeks to a maximum of $600 \mathrm{mg}$ per dose $(1800 \mathrm{mg} /$ day $)$. The study protocol was approved by the institutional review board of each institution, and the study was performed in accordance with the principles of the Helsinki Declaration of 1964 and its later amendments, as well as any relevant regulatory requirements. All patients provided written informed consent. Patients with a predicted FVC less than $50 \%$ or DLco less than $35 \%$ at baseline were classified as the advanced group.

\section{Data Collection}

Lung function (FVC and DLco) and subjective symptoms (dyspnea and cough) were assessed at 12-week intervals (i.e., 0, 12, 24, 36, and 48 weeks). Spirometry and DLco measurements were performed on the basis of the ERS/ATS guidelines, and the results were presented as percentages of normal predicted values [16-18]. Dyspnea was assessed on a scale of 1 to 5 , according to the British Medical Research Council, and the severity of cough was classified into four grades (none, mild, moderate, and severe) [19].

\section{Evaluation of Safety and Efficacy}

Safety was evaluated in patients who received pirfenidone at least once and were followed up for safety assessment. Treatment-emergent events were defined as outcomes that occurred from the first dose of pirfenidone until 7 days after discontinuation. Adverse events (AEs) were reported using preferred terms according to the Medical Dictionary for Regulatory Activities, version 21.0. Acute exacerbations of IPF were reported by investigators and were defined as events meeting all of the following criteria: (1) worsening of dyspnea within a 1-month period; (2) newly developed pulmonary infiltrates, observed on chest computed tomography images; (3) reduction of arterial oxygen tension (a decrease of at least $10 \mathrm{mmHg}$ in partial oxygen pressure compared with stable pressure); and (4) 
no evidence of precipitating factors such as infection or heart disease $[4,5]$.

Efficacy was evaluated in patients who were treated for at least 12 weeks and underwent lung function tests before and after treatment. The rate of lung function decline and absolute mean changes in predicted FVC\% and DLco\% values from baseline to 48 weeks were analyzed using the mixed-effects model repeated measurement (MMRM) to minimize the influence of missing data on calculated lung function $[20,21]$. Disease progression was determined as the absolute decline of the predicted FVC at least $10 \%$ or DLco at least $15 \%[1,22]$. Categorized changes in dyspnea and cough were defined as improved (decreased score), stable (no change), or worsened (increased score). Progression-free survival (PFS) was defined as the time from treatment initiation to the first occurrence of disease progression or death.

\section{Statistical Analysis}

Data are expressed as the mean \pm standard deviation, the mean \pm standard error (SE), or numbers with percentages, as appropriate. An independent $t$ test or Wilcoxon's rank-sum test was used for comparison of continuous variables between the groups, and Fisher's exact test or Pearson's chi-squared test was used for comparison of categorical variables between the groups. MMRM was used to analyze the rate of lung function decline and the absolute mean changes of FVC and DLco from baseline (fixed variables: visit, age, sex, and smoking status; random effect: participant's identification number). The results (rate of lung function decline and absolute mean changes from baseline) for the advanced and non-advanced groups were compared using group and groupby-visit as fixed effects, in addition to MMRM. The event-free probability was estimated using the Kaplan-Meier method, and a log-rank test was used for assessing differences between the two groups. Adjusted hazard ratios (aHRs) and 95\% confidence intervals (CIs) were generated using Cox's proportional hazards model adjusted for age and sex. All statistical analyses were performed using SAS (version 9.4, SAS Institute, Cary, NC, USA). A two-sided $p$ value less than 0.05 indicated significant differences.

\section{RESULTS}

\section{Baseline Characteristics}

Of the 258 enrolled patients, 39 were excluded from the analysis because of off-label drug use ( $n=13$ ) or missing baseline lung function data $(n=26)$. Therefore, safety was evaluated in 219 patients (39 in the advanced group and 180 in the non-advanced group) (Fig. 1). The baseline characteristics of patients are provided in Table 1 and in Table S1 in the supplementary material. The patients were mostly male $(76.3 \%)$ and ever-smokers $(68.9 \%)$, and the mean age was 67.3 years. The median time since diagnosis was 1.0 year (advanced 1.5 years vs. non-advanced 0.9 years). The median duration of treatment was 298 days, with $64.4 \%$ of the patients treated for at least 180 days. Compared with the patients in the non-advanced group, those in the advanced group had more severe subjective symptoms (cough and dyspnea), lower lung function, and more frequently received immunosuppressants at baseline. Pulmonary hypertension tended to be more prevalent in the advanced group than in the non-advanced group $(5.1 \%$ vs. $0.6 \%$, respectively; $p=0.083)$. However, there were no between-group differences in other comorbidities.

\section{Adverse Events}

A total of $86.3 \%$ of the patients experienced AEs, of which a decreased appetite (32.4\%) and a photosensitivity reaction $(13.7 \%)$ were the most frequent ones (Table 2). The incidence of AEs was similar in the advanced and non-advanced groups $(92.3 \%$ vs. $85.0 \%$, respectively; $p=0.229$; Table 2). The time-to-event analysis showed that most AEs occurred during the first 24 weeks of treatment; the Kaplan-Meier estimate of event-free probability at week 24 was $20.0 \%$ (95\% CI 14.8-25.8\%; Fig. S1 in the 


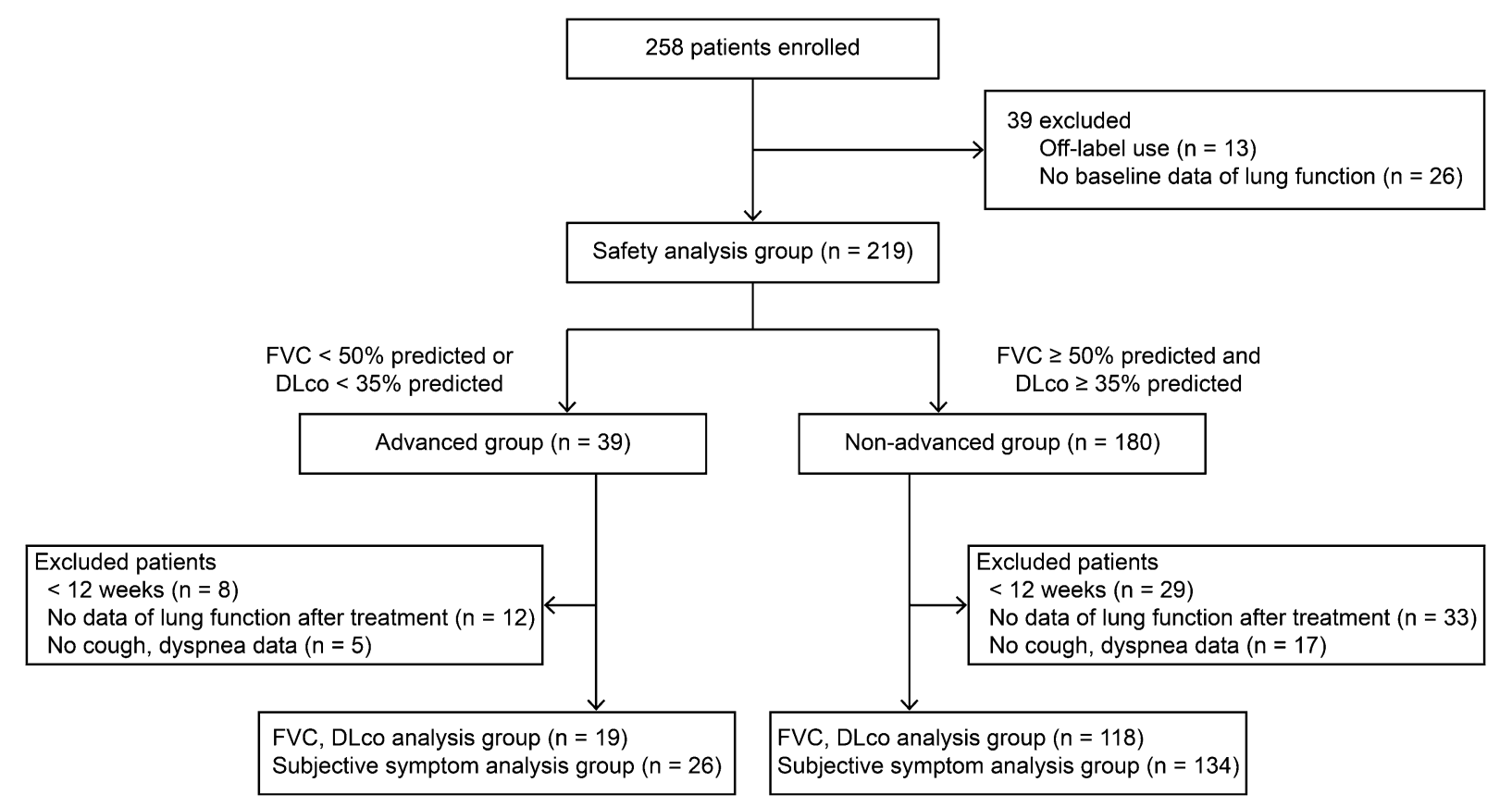

Fig. 1 Patient disposition

supplementary material). The probability of having any AEs was higher in the advanced group than in the non-advanced group (median time 6.4 vs. 9.0 weeks, respectively; aHR 1.6; $95 \%$ CI $1.1-2.3 ; p=0.020$; Fig. S1 in the supplementary material and Fig. 2). Similarly, dyspnea (incidence $18.0 \%$ vs. $6.1 \%$, respectively; aHR $3.4 ; 95 \%$ CI $1.3-8.9 ; p=0.012$ ) and progression of IPF (incidence $15.4 \%$ vs. 5.6\%, respectively; aHR 3.1; 95\% CI 1.1-8.6; $p=0.030$ ) were more prevalent in the advanced group than in the non-advanced group. However, there was no between-group difference in the risk of gastrointestinal (decreased appetite, nausea, epigastric discomfort, constipation, dyspepsia, and diarrhea) and skin-related events (photosensitivity reaction, rash, and pruritus) (Fig. 2). Serious AEs (SAEs) occurred more frequently in the advanced group than in the nonadvanced group (30.8\% vs. $15.0 \%$, respectively; $p=0.020)$. The most common SAEs were progression of IPF in the advanced group and pneumonia in the non-advanced group (Table 2).

\section{Disease-Related AEs}

Event rates for investigator-reported acute exacerbation, death, and hospitalization are summarized in Table S2 in the supplementary material. Investigator-reported acute exacerbation occurred in $4.1 \%(n=9)$ of all patients, and no between-group difference was observed (advanced group $7.7 \%$ vs. non-advanced group: $3.3 \% ; p=0.202)$. The rate of death from all causes and from IPF was 5.9\% $(n=13)$ and 3.2\% $(n=7)$, respectively. The time-to-event analysis showed a higher risk of all-cause deaths in the advanced group than in the non-advanced group (incidence $15.4 \%$ vs. $3.9 \%$, respectively; aHR $4.4 ; 95 \%$ CI $1.5-13.1 ; p=0.008$ ), but there was no significant between-group difference in the risk of IPF-related deaths (incidence $7.7 \%$ vs. $2.2 \%$, respectively; aHR $3.8 ; 95 \%$ CI $0.9-16.9$; $p=0.082$; Fig. 2 and Table 22 in the supplementary material).

All-cause hospitalization was reported in $15.1 \%$ of all patients $(n=33)$, mainly as a result of respiratory-related reasons such as pneumonia and progression of IPF. There was no significant difference in the risk of all-cause hospitalization between groups (incidence 
Table 1 Baseline characteristics of the patients in the advanced and non-advanced IPF groups

\begin{tabular}{|c|c|c|c|c|}
\hline Characteristic & Total & Advanced & Non-advanced & $p$ value \\
\hline Patients, $n$ & 219 & 39 & 180 & \\
\hline Male sex & $167(76.3)$ & $30(76.9)$ & $137(76.1)$ & 0.914 \\
\hline Ever-smoker & $151(68.9)$ & $27(69.2)$ & $124(68.9)$ & 0.967 \\
\hline Age, years & $67.3 \pm 8.0$ & $65.3 \pm 9.3$ & $67.7 \pm 7.7$ & 0.161 \\
\hline Time since diagnosis, years, median (Q1, Q3) & $1.0(0.1,3.1)$ & $1.5(0.3,3.7)$ & $0.9(0.1,2.8)$ & 0.110 \\
\hline Treatment duration, days, median (Q1, Q3) & $298(114,365)$ & $212(96,344)$ & $320(116,367)$ & 0.212 \\
\hline Follow-up duration, days, median (Q1, Q3) & $322(127,370)$ & $212(96,349)$ & $329(131,372)$ & 0.176 \\
\hline Daily dose, mg & $1081.6 \pm 430.4$ & $1110.3 \pm 422.6$ & $1075.4 \pm 432.9$ & 0.773 \\
\hline FVC, \% predicted & $65.0 \pm 15.3$ & $46.4 \pm 10.7$ & $69.0 \pm 13.0$ & $<0.001$ \\
\hline DLco, \% predicted & $52.3 \pm 16.2$ & $35.0 \pm 11.1$ & $56.0 \pm 14.6$ & $<0.001$ \\
\hline \multicolumn{5}{|l|}{ IPF treatment at baseline } \\
\hline Steroid & $7(3.2)$ & $3(7.7)$ & $4(2.2)$ & 0.553 \\
\hline Immunosuppressant & $2(0.9)$ & $2(5.1)$ & $0(0.0)$ & 0.031 \\
\hline Cysteine derivative & $25(11.4)$ & $3(7.7)$ & $22(12.2)$ & 0.582 \\
\hline Oxygen therapy at baseline & $5(2.3)$ & $2(5.1)$ & $3(1.7)$ & 0.217 \\
\hline Comorbidities & $178(81.3)$ & $34(87.2)$ & $144(80.0)$ & 0.297 \\
\hline Hypertension & $60(27.4)$ & $12(30.8)$ & $48(26.7)$ & 0.603 \\
\hline Diabetes mellitus & $60(27.4)$ & $12(30.8)$ & $48(26.7)$ & 0.603 \\
\hline GERD & $33(15.1)$ & $6(15.4)$ & $27(15.0)$ & 0.952 \\
\hline Hyperlipidemia & $25(11.4)$ & $5(12.8)$ & $20(11.1)$ & 0.782 \\
\hline Emphysema & $8(3.7)$ & $2(5.1)$ & $6(3.3)$ & 0.635 \\
\hline COPD & $6(2.7)$ & $1(2.6)$ & $5(2.8)$ & 1.000 \\
\hline Pulmonary hypertension & $3(1.7)$ & $2(5.1)$ & $1(0.6)$ & 0.083 \\
\hline
\end{tabular}

Data are presented as the mean \pm standard deviation or number (\%), unless otherwise specified

Q1 quartile 1 (25th percentile), Q3 quartile 3 (75th percentile), GERD gastroesophageal reflux disease, COPD chronic obstructive pulmonary disease, $I P F$ idiopathic pulmonary fibrosis, $F V C$ forced vital capacity, $D L c o$ diffusing capacity of the lung for carbon monoxide

$20.5 \%$ vs. $13.9 \%$; aHR $1.9 ; 95 \%$ CI $0.9-4.3$; $p=0.108)$. However, the advanced group showed a greater risk of respiratory hospitalization than did the non-advanced group (incidence $20.5 \%$ vs. $11.1 \%$, respectively; aHR 2.5 ; 95\% CI 1.1-5.8; $p=0.031$; Fig. 2 and Table S2 in the supplementary material). There was no significant difference in progression-free survival between the groups (aHR 1.4; 95\% CI 0.7-2.8; $p=0.381$; Fig. 2).

\section{Treatment Discontinuation}

Treatment was prematurely discontinued in $54.3 \%$ of the patients (Table 3 ). The advanced 
Table 2 Adverse events (frequency at least 3\%) in the advanced and non-advanced IPF groups

\begin{tabular}{|c|c|c|c|c|}
\hline Characteristic & Total & Advanced & Non-advanced & $p$ value \\
\hline Patients, $n$ & 219 & 39 & 180 & \\
\hline Adverse events & $189(86.3)$ & $36(92.3)$ & $153(85.0)$ & 0.229 \\
\hline Decreased appetite & $71(32.4)$ & $13(33.3)$ & $58(32.2)$ & 0.893 \\
\hline Photosensitivity reaction & $30(13.7)$ & $6(15.4)$ & $24(13.3)$ & 0.736 \\
\hline Rash & $25(11.4)$ & $2(5.1)$ & $23(12.8)$ & 0.266 \\
\hline Nausea & $24(11.0)$ & $6(15.4)$ & $18(10.0)$ & 0.394 \\
\hline Pruritus & $24(11.0)$ & $1(2.6)$ & $23(12.8)$ & 0.087 \\
\hline Epigastric discomfort & $22(10.1)$ & $4(10.3)$ & $18(10.0)$ & 1.000 \\
\hline Cough & $21(9.6)$ & $2(5.1)$ & $19(10.6)$ & 0.383 \\
\hline Pneumonia & $19(8.7)$ & $6(15.4)$ & $13(7.2)$ & 0.117 \\
\hline Dyspnea & $18(8.2)$ & $7(18.0)$ & $11(6.1)$ & 0.023 \\
\hline Progression of $\mathrm{IPF}^{\mathrm{a}}$ & $16(7.3)$ & $6(15.4)$ & $10(5.6)$ & 0.044 \\
\hline Productive cough & $15(6.9)$ & $4(10.3)$ & $11(6.1)$ & 0.314 \\
\hline Constipation & $13(5.9)$ & $3(7.7)$ & $10(5.6)$ & 0.707 \\
\hline Fatigue & $11(5.0)$ & $4(10.3)$ & $7(3.9)$ & 0.111 \\
\hline Asthenia & $10(4.6)$ & $1(2.6)$ & $9(5.0)$ & 1.000 \\
\hline Dizziness & $10(4.6)$ & $1(2.6)$ & $10(5.6)$ & 0.694 \\
\hline Upper respiratory tract infection & $9(4.1)$ & $0(0.0)$ & $9(5.0)$ & 0.367 \\
\hline Dyspepsia & $8(3.7)$ & $2(5.1)$ & $6(3.3)$ & 0.635 \\
\hline Diarrhea & $8(3.7)$ & $2(5.1)$ & $6(3.3)$ & 0.635 \\
\hline Abnormal liver function test & $8(3.7)$ & $1(2.6)$ & $7(3.9)$ & 1.000 \\
\hline Serious adverse events ${ }^{\mathrm{b}}$ & $39(17.8)$ & $12(30.8)$ & $27(15.0)$ & 0.020 \\
\hline Pneumonia & $15(6.9)$ & $3(7.7)$ & $12(6.7)$ & 0.734 \\
\hline Progression of $\mathrm{IPF}^{\mathrm{c}}$ & $13(5.9)$ & $4(10.3)$ & $9(5.0)$ & 0.255 \\
\hline Adverse drug reactions ${ }^{\mathrm{d}}$ & $151(69.0)$ & $27(69.2)$ & $124(68.9)$ & 0.967 \\
\hline
\end{tabular}

Data are presented as the number (\%), unless otherwise specified

$I P F$ idiopathic pulmonary fibrosis

${ }^{a}$ Includes acute exacerbations of IPF $(n=9)$

b A serious adverse event was defined as an adverse event that was fatal or life-threatening, required prolonged hospitalization, was associated with a congenital anomaly, or was deemed to be serious for any other reason

c These were the events meeting one of the criteria for serious adverse events among the adverse events of progression of IPF

d A summary of adverse drug reactions with a frequency of at least 3\% is presented in Table S5 in the supplementary material 


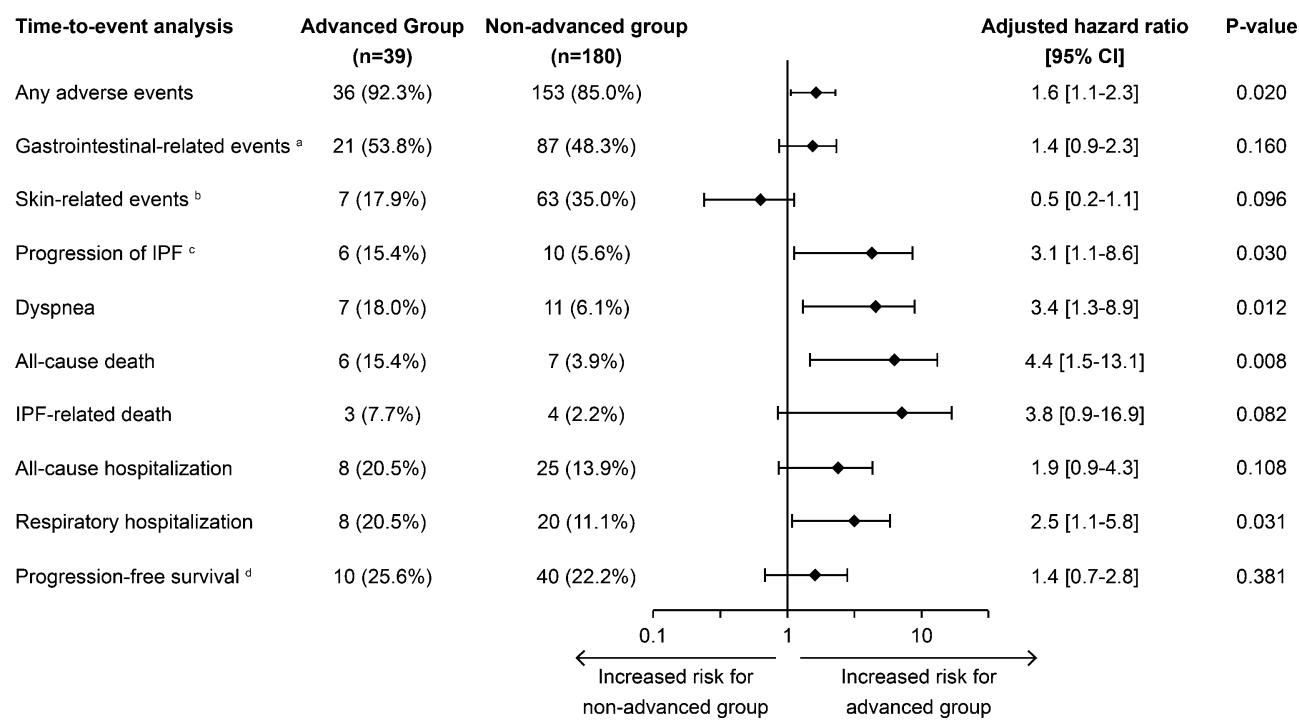

Fig. 2 Forest plot demonstrating treatment-emergent adverse events and progression-free survival in the advanced and non-advanced IPF groups. Hazard ratios between the advanced and non-advanced groups were compared using Cox's proportional hazards model adjusted for age and sex. IPF idiopathic pulmonary fibrosis, CI confidence interval. " Included decreased appetite,

group had a higher rate of discontinuation than did the non-advanced group ( $74.4 \%$ vs. $50.0 \%$, respectively; $p=0.006$ ). The most common reason for discontinuation was AEs (22.8\%), followed by patient request (16.0\%), no revisit $(9.1 \%)$, progression of IPF $(5.0 \%)$, and insufficient efficacy (1.4\%). There were no betweengroup differences in the reasons for discontinuation, except progression of IPF (advanced group $12.8 \%$ vs. non-advanced group 3.3\%; $p=0.028$ ). Of 189 patients who experienced AEs, $55.6 \%$ continued treatment without dose interruption, and no between-group difference was observed (Table S3 in the supplementary material).

\section{Changes in Lung Function}

Changes in lung function from baseline to 48 weeks were evaluated in 137 patients (19 in the advanced group and 118 in the non-advanced group). The patients were mostly male $(74.5 \%)$ and ever-smokers (70.1\%), with a median treatment duration of 346 days (range nausea, epigastric discomfort, constipation, dyspepsia, and diarrhea; 'bncluded photosensitivity reaction, rash, and pruritus; ' Included exacerbations and acute exacerbations of IPF; ${ }^{\mathrm{d}}$ Progression-free survival was defined as the time from treatment initiation to the occurrence of death or disease progression (determined as an absolute decline of the predicted FVC at least $10 \%$ or DLco at least $15 \%$ )

99-889 days). The baseline characteristics were comparable between the groups, except for lung function (Table S4 in the supplementary material). The adjusted absolute change $( \pm$ SE) from baseline to 48 weeks in the predicted FVC was $-3.1 \pm 0.8 \%$, and that in the predicted DLco was $-5.0 \pm 1.2 \%$ (Fig. $3 a, b$ ). The adjusted decline rate over 48 weeks in the predicted FVC was $-4.3 \pm 1.3 \%$, and that in the predicted DLco was $-4.4 \pm 1.7 \%$ (Fig. 3c, d). Overall, no between-group differences were observed in the mean FVC and DLco changes and in the adjusted rates of lung function decline, which indicated a consistent treatment effect, irrespective of disease severity. The proportion of patients who experienced an FVC decline of at least $10 \%$ or a DLco decline of at least $15 \%$ was $13.1 \%$ and $14.6 \%$, respectively. No significant differences were observed in terms of categorical changes in lung function between the groups (Fig. S2a, b in the supplementary material). 
Table 3 Reasons for premature discontinuation of the treatment in the advanced and non-advanced IPF groups

\begin{tabular}{|c|c|c|c|c|}
\hline Reasons for discontinuation & Total & Advanced & Non-advanced & $p$ value \\
\hline Total patients, $n$ & 219 & 39 & 180 & \\
\hline Discontinued patients & $119(54.3)$ & $29(74.4)$ & $90(50.0)$ & 0.006 \\
\hline Adverse event & $50(22.8)$ & $8(20.5)$ & $42(23.3)$ & 0.704 \\
\hline Decreased appetite & $10(4.6)$ & $1(2.6)$ & $9(5.0)$ & 1.000 \\
\hline Photosensitivity reaction & $9(4.1)$ & $1(2.6)$ & $8(4.4)$ & 1.000 \\
\hline Rash & $6(2.7)$ & $0(0.0)$ & $6(3.3)$ & 0.594 \\
\hline Cough & $4(1.8)$ & $0(0.0)$ & $4(2.2)$ & 1.000 \\
\hline Dyspnea & $4(1.8)$ & $1(2.6)$ & $3(1.7)$ & 0.546 \\
\hline Epigastric discomfort & $4(1.8)$ & $1(2.6)$ & $3(1.7)$ & 0.546 \\
\hline Nausea & $4(1.8)$ & $0(0.0)$ & $4(2.2)$ & 1.000 \\
\hline Pneumonia & $4(1.8)$ & $0(0.0)$ & $4(2.2)$ & 1.000 \\
\hline Abdominal pain & $2(0.9)$ & $0(0.0)$ & $2(1.1)$ & 1.000 \\
\hline Dizziness & $2(0.9)$ & $0(0.0)$ & $2(1.1)$ & 1.000 \\
\hline Myocardial infarction & $2(0.9)$ & $1(2.6)$ & $1(0.6)$ & 0.325 \\
\hline Pruritus & $2(0.9)$ & $0(0.0)$ & $2(1.1)$ & 1.000 \\
\hline Pyrexia & $2(0.9)$ & $1(2.6)$ & $1(0.6)$ & 0.325 \\
\hline Patient request ${ }^{a}$ & $35(16.0)$ & $10(25.6)$ & $25(13.9)$ & 0.069 \\
\hline Before October 2, 2015 & $28(12.8)$ & $8(20.5)$ & $20(11.1)$ & 0.118 \\
\hline After October 2, 2015 & $7(3.2)$ & $2(5.1)$ & $5(2.8)$ & 0.611 \\
\hline No revisit & $20(9.1)$ & $5(12.8)$ & $15(8.3)$ & 0.365 \\
\hline Progression of $\mathrm{IPF}^{\mathrm{b}}$ & $11(5.0)$ & $5(12.8)$ & $6(3.3)$ & 0.028 \\
\hline Insufficient efficacy & $3(1.4)$ & $1(2.6)$ & $2(1.1)$ & 0.446 \\
\hline
\end{tabular}

Data are presented as the number (\%), unless otherwise specified

$I P F$ idiopathic pulmonary fibrosis

${ }^{a}$ The medication cost was not covered by the National Health Insurance Service in Korea until October 2015

b Includes acute exacerbations of IPF $(n=6)$

\section{Changes in Subjective Symptoms}

Of 160 patients (26 in the advanced group and 134 in the non-advanced group) who were treated for at least 12 weeks and whose subjective symptoms were assessed before and after treatment, $8.8 \%$ experienced improvement, $70.6 \%$ experienced stabilization, and $20.6 \%$ experienced a worsening of dyspnea (Fig. S2c in the supplementary material). The proportion of patients with improved, stable, and worsened cough was $11.9 \%, 68.8 \%$, and $19.4 \%$, respectively (Fig. S2d in the supplementary material). There were no significant between-group differences in categorical changes of the subjective symptoms. 

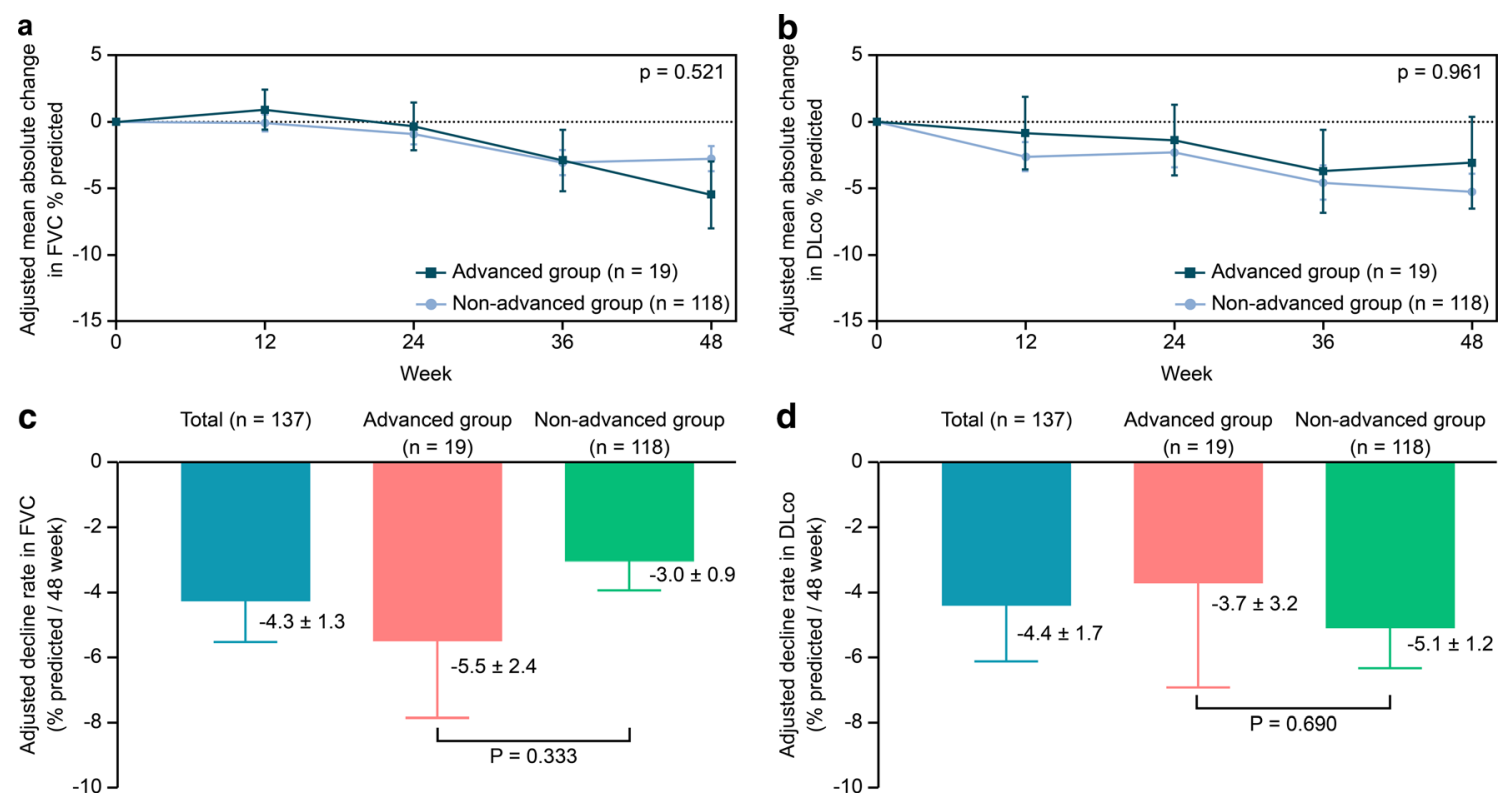

Fig. 3 Comparison of absolute changes and annual decline rates in the predicted FVC\% and DLco\%. a Adjusted mean absolute change in the predicted FVC\%. b Adjusted mean absolute change in the predicted DLco\%. c Adjusted decline rate of FVC (\% predicted/ 48 weeks). d Adjusted decline rate of DLco (\% predicted/ 48 weeks). Values are the mean \pm SE. The mixed-effects model repeated measurement (MMRM) was used to analyze the rate of decline of lung function and the mean

\section{DISCUSSION}

This was the first nationwide study conducted to obtain prospective real-world data on the treatment effect of pirfenidone in Korean patients with IPF. We conducted a post hoc analysis of the data collected to compare the therapeutic effects of pirfenidone in patients with advanced IPF and in those with non-advanced IPF. Our study demonstrated that pirfenidone had an acceptable safety profile and showed a consistent efficacy in terms of stabilizing lung function and subjective symptoms, regardless of IPF disease severity.

The types of AEs observed were similar in the advanced and non-advanced IPF groups and were consistent with the known safety profile of pirfenidone. Most AEs occurred within the first 6 months of treatment, and the frequency of AEs was reduced over time. The multivariate

changes of FVC and DLco from baseline, with the visit, age, sex, and smoking status as fixed effects and participant's identification number as the random effect. To compare the results between the advanced and nonadvanced groups, the group and the group-by-visit were used as a fixed effect, in addition to MMRM. FVC forced vital capacity, DLco diffusing capacity of the lung for carbon monoxide

Cox analysis showed that the advanced group had a greater risk of experiencing AEs than did the non-advanced group. This difference appeared to be due to an increased risk of progression of IPF and dyspnea in the advanced group, which could be attributed to the severity of the disease. However, there was no significant difference in the risk of drug-related gastrointestinal and skin-related events between the groups. Most AEs were mild or moderate in severity and reversible upon treatment interruption. The treatment discontinuation rate was higher in the advanced group than in the non-advanced group, similar to previous studies $[15,23,24]$. However, the proportion of patients who discontinued treatment as a result of AEs (22.8\%) was similar in both groups and was not different from that reported in other observational studies (18.4-26.7\%) [11-15, 24]. In this study, the rate of discontinuation by 
patient request $(16.0 \%)$ was relatively higher than that reported in a Japanese post-marketing study $(5.3 \%)$ [24], but our finding should be interpreted with caution because the medication cost was not covered by the National Health Insurance Service in Korea until October 2015. Importantly, $12.8 \%$ of all patients discontinued treatment because of economic reasons before October 2015; however, the rate of discontinuation decreased (3.2\%) thereafter.

Acute exacerbation of IPF is a common and fatal complication, especially in patients with progressive disease, with a 1-year incidence of $5-14.2 \%[25,26]$. In our study, the incidence of acute exacerbation was lower $(4.1 \%)$ than that reported in previous studies $[25,26]$, and there was no significant difference in the risk of acute exacerbations between the advanced and nonadvanced groups (Fig. S3 in the supplementary material). It has been reported that patients with lower lung function are at a greater risk of acute exacerbation of IPF [25]. Collectively, our study results suggest that pirfenidone treatment might be more beneficial in preventing acute exacerbation in patients with advanced IPF who are at a higher risk of such an event than in those with non-advanced IPF. A post hoc analysis of ASCEND (NCT01366209) and CAPACITY trials (NCT00287716 and NCT00287729) also showed a significantly lower risk of respiratory hospitalization in patients with advanced IPF (predicted FVC less than $50 \%$ or $\mathrm{DL}_{\mathrm{CO}}$ less than $30 \%$ ) than placebo-treated patients (HR 0.4; 95\% CI $0.2-0.9 ; p=0.021)$; IPF was the most frequent reason for respiratory hospitalization [27].

It has been reported that acute respiratory events, which are mostly caused by acute exacerbation and pulmonary infections, are common in patients with IPF and lead to hospitalization [25], which is associated with a decreased survival of patients with IPF $[9,28,29]$. In this study, of the patients who died during the study $(n=13)$, approximately half $(46.2 \%, n=6)$ underwent respiratory hospitalization, and the advanced group showed a greater risk of respiratory hospitalization, mainly due to pneumonia and progression of IPF.
The baseline disease severity, the time since diagnosis, and the presence of comorbidities have been associated with a decreased survival of patients with IPF $[30,31]$. In our study, while the rate of all-cause deaths was higher in the advanced group than in the non-advanced group $(15.4 \%$ vs. $3.9 \%$, respectively; $p=0.014)$, no significant between-group difference was observed in the rates of IPF-related deaths $(7.7 \%$ vs. $2.2 \%$, respectively; $p=0.109$ ). Non-IPF-related deaths frequently occurred in the population of patients with IPF, the majority of which are elderly people [2]; therefore, IPF-related deaths could be a more accurate measure of the treatment efficacy. Thus, our results may provide more accurate evidence of the clinical benefit of pirfenidone in patients with advanced IPF; however, the number of patients was too small for conclusions to be drawn.

The absolute decline and annual decline rate in FVC in patients with advanced IPF were comparable to those in patients with non-advanced IPF; a similar trend was observed for DLco. The rates of disease progression, subjective symptoms, and PFS were also similar between the groups. These results suggested that pirfenidone-treated patients with advanced IPF might experience a similar degree of efficacy as that in patients with non-advanced IPF. Similar findings were also reported in recent studies [11-15, 23]. Post hoc analyses of the RECAP trial reported that the rate of the annual FVC decline in patients with advanced IPF (predicted FVC less than 50\% or DLco less than $35 \%)$ was similar to that in patients with less advanced IPF (3.8\% vs. 3.9\%, respectively) [15]. In the INPULSIS-ON study, in which patients were treated with nintedanib, the absolute changes in FVC from baseline to week 48 were similar in patients with predicted FVC values at most $50 \%$ and greater than 50\% (-62.3 vs. $-87.9 \mathrm{~mL}$, respectively) [23]. A retrospective study of pirfenidone including 43 patients with severe IPF (predicted FVC less than $50 \%$ or DLco less than 35\%) conducted by Tzouvelekis et al. [14] also showed that the rate of FVC decline at 6 months of treatment was not significantly different between groups with predicted FVC less than 50\% and at least 50\% 
$(4.7 \pm 8.9$ vs. $-0.6 \pm 11.8 \%, \quad$ respectively; $p=0.2)$.

There are several limitations to this study. First, the number of patients in the advanced group was smaller than that in the non-advanced group. In order to overcome this limitation, we analyzed the data from various perspectives, and our study showed consistent trends in the treatment effects across different parameters such as lung function change, subjective symptoms change, hospitalization, mortality, and progression-free survival between the two groups, with the results being highly consistent with those of previous studies. Second, the absence of a control group limited the extent of a meaningful interpretation of the results. However, previous randomized, placebo-controlled trials have demonstrated that pirfenidone treatment shows sufficient effects in patients with IPF. Third, the IPF diagnosis was performed by participating investigators, which could have led to a confirmation bias in some patients. However, the demographic and clinical features were comparable to those of other studies. Fourth, it should be also recognized that the results of this study are limited by the relatively lower dosage used (at most $1800 \mathrm{mg} /$ day), and the minimum treatment duration of 12 weeks for efficacy evaluation. However, considering the standard body weight in Korea, the weight-adjusted dose in this study could be comparable to that in European countries and the USA. In addition, a significant treatment effect of pirfenidone versus placebo was evident from week 12 in CAPACITY phase 3 trials. Lastly, a relatively high proportion of patients discontinued treatment as a result of reimbursement restrictions. As a result, the treatment discontinuation may have been overestimated.

\section{CONCLUSIONS}

The results of this study indicated that treatment with pirfenidone in a real-world setting produced an acceptable safety profile and a consistent therapeutic effect in patients with IPF in terms of stabilizing lung function and subjective symptoms, regardless of the disease severity. Additional studies may be needed to further confirm these findings.

\section{ACKNOWLEDGEMENTS}

We thank all patients and participating staff for their cooperation in this study.

Funding. This study was funded by Ildong Pharmaceutical Co., Ltd., which collaborated with the authors in the design of the study and collection, analysis, and interpretation of the data. Ildong Pharmaceutical Co., Ltd. also funded the journal's rapid service and open access fees.

Authorship. All named authors meet the International Committee of Medical Journal Editors (ICMJE) criteria for authorship for this article, take responsibility for the integrity of the work as a whole, and have given their approval for this version to be published. JW contributed to the conception and design of the study, analysis and interpretation of the data, and drafting and revising the manuscript. MP and MS contributed to data collection and interpretation. IJ, HB, YW, JS, ST, YS, and YJ contributed to data collection. MP and MS conceived and designed the study and helped revise the manuscript. All authors read and approved the final manuscript.

Medical Writing and Editorial Assistance. Medical writing and editorial assistance was provided by Mi-Sun Park of Ildong Pharmaceutical Co., Ltd. while writing this article. We are grateful to Editage (www.editage.com) for English language editing and publication support.

Disclosures. Man Pyo Chung, Moo Suk Park, In Jae Oh, Heung Bum Lee, Young Whan Kim, Jong Sun Park, Soo Taek Uh, Yun Seong Kim, Yangjin Jegal, and Jin Woo Song have nothing to disclose.

Compliance with Ethics Guidelines. The study protocol was approved by the institutional review board of each institution [Asan 
Medical Center (2014-0810); Samsung Medical Center (SMC PMS2014-012); Yonsei University Health System (4-2014-0248); Chonnam National University Hwasun Hospital (CNUHH2014-067); Chonbuk National University Hospital (CUH PMS2014-007); Seoul National University Hospital (H-1405-033-579); Seoul National University Bundang Hospital (B-1407/ 260-202); Soonchunhyang University Seoul Hospital (SCHUH PMS2014-014); Pusan National University Yangsan Hospital (06-2014012); and Ulsan University Hospital (UUH PMS2014-018)]. The study was conducted in accordance with the principles of the Helsinki Declaration of 1964 and its later amendments, as well as any relevant regulatory requirements. All patients provided written informed consent.

Data availability. The datasets generated and/or analyzed during the current study are not publicly available to protect individual patient confidentiality, but are available from the corresponding author on reasonable request with the permission of Ildong Pharmaceutical Co., Ltd.

Open Access. This article is licensed under a Creative Commons Attribution-NonCommercial 4.0 International License, which permits any non-commercial use, sharing, adaptation, distribution and reproduction in any medium or format, as long as you give appropriate credit to the original author(s) and the source, provide a link to the Creative Commons licence, and indicate if changes were made. The images or other third party material in this article are included in the article's Creative Commons licence, unless indicated otherwise in a credit line to the material. If material is not included in the article's Creative Commons licence and your intended use is not permitted by statutory regulation or exceeds the permitted use, you will need to obtain permission directly from the copyright holder.To view a copy of this licence, visit http://creativecommons.org/licenses/by$\mathrm{nc} / 4.0 /$.

\section{REFERENCES}

1. Raghu G, Collard HR, Egan JJ, et al. An official ATS/ ERS/JRS/ALAT statement: idiopathic pulmonary fibrosis: evidence-based guidelines for diagnosis and management. Am J Respir Crit Care Med. 2011;183:788-824.

2. Ley B, Collard HR, King TE Jr. Clinical course and prediction of survival in idiopathic pulmonary fibrosis. Am J Respir Crit Care Med. 2011;183: 431-40.

3. Schaefer CJ, Ruhrmund DW, Pan L, Seiwert SD, Kossen K. Antifibrotic activities of pirfenidone in animal models. Eur Respir Rev. 2011;20:85-97.

4. Azuma A, Nukiwa T, Tsuboi E, et al. Double-blind, placebo-controlled trial of pirfenidone in patients with idiopathic pulmonary fibrosis. Am J Respir Crit Care Med. 2005;171:1040-7.

5. Taniguchi H, Ebina M, Kondoh Y, et al. Pirfenidone in idiopathic pulmonary fibrosis. Eur Respir J. 2010;35:821-9.

6. Noble PW, Albera C, Bradford WZ, et al. Pirfenidone in patients with idiopathic pulmonary fibrosis (CAPACITY): two randomised trials. Lancet. 2011;377:1760-9.

7. King TE, Bradford WZ, Castro-Bernardini S, et al. A phase 3 trial of pirfenidone in patients with idiopathic pulmonary fibrosis. N Engl J Med. 2014;370: 2083-92.

8. Noble PW, Albera C, Bradford WZ, et al. Pirfenidone for idiopathic pulmonary fibrosis: analysis of pooled data from three multinational phase 3 trials. Eur Respir J. 2016;47:243-53.

9. Ley B, Swigris J, Day BM, et al. Pirfenidone reduces respiratory-related hospitalizations in idiopathic pulmonary fibrosis. Am J Respir Crit Care Med. 2017;196:756-61.

10. Albera C, Costabel U, Fagan EA, et al. Efficacy of pirfenidone in patients with idiopathic pulmonary fibrosis with more preserved lung function. Eur Respir J. 2016;48:843-51.

11. Okuda R, Hagiwara E, Baba T, Kitamura H, Kato T, Ogura T. Safety and efficacy of pirfenidone in idiopathic pulmonary fibrosis in clinical practice. Respir Med. 2013;107:1431-7.

12. Harari S, Caminati A, Albera C, et al. Efficacy of pirfenidone for idiopathic pulmonary fibrosis: an Italian real life study. Respir Med. 2015;109:904-13. 
13. Yoon HY, Kim DS, Song JW. Efficacy and safety of pirfenidone in advanced idiopathic pulmonary fibrosis. Respiration. 2019;97:242-51.

14. Tzouvelekis A, Ntolios P, Karampitsakos T, et al. Safety and efficacy of pirfenidone in severe idiopathic pulmonary fibrosis: a real-world observational study. Pulm Pharmacol Ther. 2017;46:48-53.

15. Costabel U, Albera C, Glassberg MK, et al. Effect of pirfenidone in patients with more advanced idiopathic pulmonary fibrosis. Respir Res. 2019;20:55.

16. Wanger J, Clausen JL, Coates A, et al. Standardization of the measurement of lung volumes. Eur Respir J. 2005;26:511-22.

17. Miller MR, Hankinson J, Brusasco V, et al. Standardisation of spirometry. Eur Respir J. 2005;26: 319-38.

18. Macintyre N, Crapo RO, Viegi G, et al. Standardisation of the single-breath determination of carbon monoxide uptake in the lung. Eur Respir J. 2005;26: 720-35.

19. Bestall J, Paul E, Garrod R, Garnham R, Jones P, Wedzicha J. Usefulness of the Medical Research Council (MRC) dyspnea scale as a measure of disability in patients with chronic obstructive pulmonary disease. Thorax. 1999;54:581-6.

20. Mallinckrodt CH, Clark WS, David SR. Accounting for dropout bias using mixed-effects models. J Biopharm Stat. 2001;11:9-21.

21. Siddiqui O, Hung HM, O’Neill R. MMRM vs. LOCF: a comprehensive comparison based on simulation study and 25 NDA datasets. J Biopharm Stat. 2009;19:227-46.

22. Richeldi L, Ryerson CJ, Lee JS, et al. Relative versus absolute change in forced vital capacity in idiopathic pulmonary fibrosis. Thorax. 2012;67:407-11.

23. Wuyts WA, Kolb M, Stowasser S, Stansen W, Huggins JT, Raghu G. First data on efficacy and safety of nintedanib in patients with idiopathic pulmonary fibrosis and forced vital capacity of $\leq 50 \%$ of predicted value. Lung. 2016;194:739-43.

24. Ogura T, Azuma A, Inoue Y, et al. All-case postmarketing surveillance of 1371 patients treated with pirfenidone for idiopathic pulmonary fibrosis. Respir Investig. 2015;53:232-41.

25. Song JW, Hong SB, Lim CM, Koh Y, Kim DS. Acute exacerbation of idiopathic pulmonary fibrosis: incidence, risk factors and outcome. Eur Respir J. 2011;37:356-63.

26. Kondoh $\mathrm{Y}$, Taniguchi H, Katsuta T, et al. Risk factors of acute exacerbation in idiopathic pulmonary fibrosis. Sarcoidosis Vasc Diffuse Lung Dis. 2010;27: 103-10.

27. Nathan SD, Costabel U, Albera C, et al. Pirfenidone in patients with idiopathic pulmonary fibrosis and more advanced lung function impairment. Respir Med. 2019;153:44-51.

28. Brown AW, Fischer CP, Shlobin OA, et al. Outcomes after hospitalization in idiopathic pulmonary fibrosis: a cohort study. Chest. 2015;147:173-9.

29. Durheim MT, Collard HR, Roberts RS, et al. Association of hospital admission and forced vital capacity endpoints with survival in patients with idiopathic pulmonary fibrosis: analysis of a pooled cohort from three clinical trials. Lancet Respir Med. 2015;3:388-96.

30. Margaritopoulos GA, Antoniou KM, Wells AU. Comorbidities in interstitial lung diseases. Eur Respir Rev. 2017;26:160027.

31. Mura M, Porretta MA, Bargagli E, et al. Predicting survival in newly diagnosed idiopathic pulmonary fibrosis: a 3-year prospective study. Eur Respir J. 2012;40:101-9. 